\title{
Calibration of the operative cosmic ray detector at Marambio Base in the Antarctic Peninsula
}

\author{
Noelia Santos', Sergio Dasso ${ }^{1,2,3}$, Adriana M. Gulisano ${ }^{2,3,4}$, Omar Areso ${ }^{2}$ and \\ Matías Pereira ${ }^{2}$, for the LAGO collaboration \\ Correspondence \\ ${ }^{1}$ Facultad de Ciencias Exactas y Naturales (FCEN), Departamento de Ciencias de la Atmósfera y los Océanos \\ (DCAO), Grupo LAMP, Universidad de Buenos Aires (UBA), Argentina, nsantos@at.fcen.uba.ar \\ ${ }^{2}$ Consejo Nacional de Investigaciones Científicas y Técnicas (CONICET), Instituto de Astronomía y Física del \\ Espacio (IAFE), Grupo LAMP, Universidad de Buenos Aires, sdasso@conicet.gov.ar \\ ${ }^{3}$ Facultad de Ciencias Exactas y Naturales (FCEN), Departamento de Física (DF), Grupo LAMP, Universidad de \\ Buenos Aires (UBA), Argentina \\ ${ }^{4}$ Instituto Antártico Argentino, Dirección Nacional del Antártico, Grupo LAMP, Universidad de Buenos Aires (UBA), \\ San Martín, Argentina \\ ${ }^{5}$ http://lagoproject.net/collab.html
}

\section{OPEN ACCESS}

This work is published under the Creative Commons Attribution 4.0 International licence (CC BY 4.0) Please note that individual, appropriately marked parts of the work may be excluded from the licence mentioned or may be subject to other copyright conditions.

If such thirdparty mans. If such thirdparty material is not under the Creative Commons licens, any copying, editing or public reproduction is only permitted with the prior consent of the respective copyright owner or on the basis of relevan legal authorization regulations.

\section{Keywords}

cosmic ray detector; water Cherenkov detector; space weather

\begin{abstract}
During 2019 an Antarctic Space Weather Laboratory was deployed at Marambio base in the Antarctic Peninsula. The main instrument installed was a cosmic ray detector based on water Cherenkov radiation (WCD). This detector is the first permanent Antarctic node of the LAGO (Latin American Giant Observatory) Collaboration. Long-term calibrated observations of the WCD will be presented here. Finally, the global galactic cosmic rays variability observed with the WCD will be compared with observations of a neutron monitor with similar rigidity cut off than the Marambio site.
\end{abstract}

\section{Introduction}

The flux of low-energy galactic cosmic rays (GCRs) is modulated by transient solar events. Thus, particle detectors at ground level that record temporal variations of the flux of secondary cosmic rays (SCRs) could provide important information for Space Weather studies. For instance, Forbush Decreases (FDs) are observed by neutron monitors (NMs) as well as by water Cherenkov detectors (WCDs) (The Pierre Auger collaboration, 2011). WCDs operating in a counting mode are highly sensitive to FDs (e.g., The Pierre Auger collaboration 2011, and Dasso \& Asorey 2012). WCDs have the advantage of being able to discriminate SCRs by their deposited energy into the detector. They are robust, low-cost (Allekotte et al. 2007) and last but not least, easy maintenance and eco-friendly.

We will present the WCD set up at the Marambio Argentinian Base in the Antarctic Peninsula for space weather studies that is part of the LAGO network (http://lagoproject.net/, last accessed April 9, 2021; Asorey \& Dasso 2015; Dasso at al. 2015; Sidelnik 2016). The advantage of having a detector in Antarctica is that low energy particles can reach the site due to its low magnetic rigidity cut off. This is one of a few WCDs at the Antarctic soil that is measuring operationally since its installation in March 2019, with IceTop and IceCube similar detectors (e.g., Karle 2008). 
In Section 2 we describe the detector and its calibration. In Section 3 we present the first observations of the pressure-corrected count rate. In Section 4 we compare WCD count rate observations with the ones from Oulu NM. Finally, in Section 5 we present a summary and conclusions.

\section{A water Cherenkov detector for space weather studies}

An Antarctic Space Weather Laboratory was deployed by the LAMP group (Laboratorio Argentino de Meteorología del esPacio, http://spaceweather.at.fcen.uba.ar/2/lamp, last accessed April 9, 2021) at Marambio in the Antarctic Peninsula, between January and March 2019. Marambio is an Argentinian Antarctic base located at 64.24S-56.62W and $196 \mathrm{~m}$ a.s.l. Its vertical geomagnetic rigidity cut off is 2.32 GV (Masías-Meza \& Dasso 2014). The main instrument installed was a WCD called Neurus. The laboratory also has a magnetometer, a GPS system to make the time stamp of observations, a meteorological station and a telemetry system which provides 5-minutes real-time monitoring at the serves of the group at Buenos Aires.

This is the first permanent Antarctic detector of the LAGO Collaboration. The LAGO Project (Latin American Giant Observatory) is an extended Astroparticle Observatory based on WCDs spanning over Latin America (Asorey \& Dasso 2015). It is mainly oriented to basic research in three branches of Astroparticle Physics: the Extreme Universe, Atmospheric Radiation at ground level and Space Weather Phenomena. The LAGO Space Weather program is directed towards the study of how the variations of the flux of SCRs at ground level are linked with the heliospheric and geomagnetic modulations (Asorey \& Dasso 2015).

The Neurus WCD consists of a stainless steel cylindrical tank (diameter $=0.96 \mathrm{~m}$, height $=$ $1.20 \mathrm{~m}$ ) which is full of purified water. When charged particles enter the detector with a speed greater than the speed of light in water they produce Cherenkov radiation which is detected by a photomultiplier tube (PMT). An internal coating made from Tyvek ${ }^{\circledR}$ assures the reflection and diffusion of the Cherenkov photons inside the tank. Through simulations it is known that WCDs enable the possibility of measure the muonic and electromagnetic components of the Extensive Air Showers (EAS), essentially dominated by $\mu \pm$, e \pm and $\gamma$ (e.g., Sarmiento-Cano et al. 2019). Pictures of the detector can be seen in the left panel of figure 1.
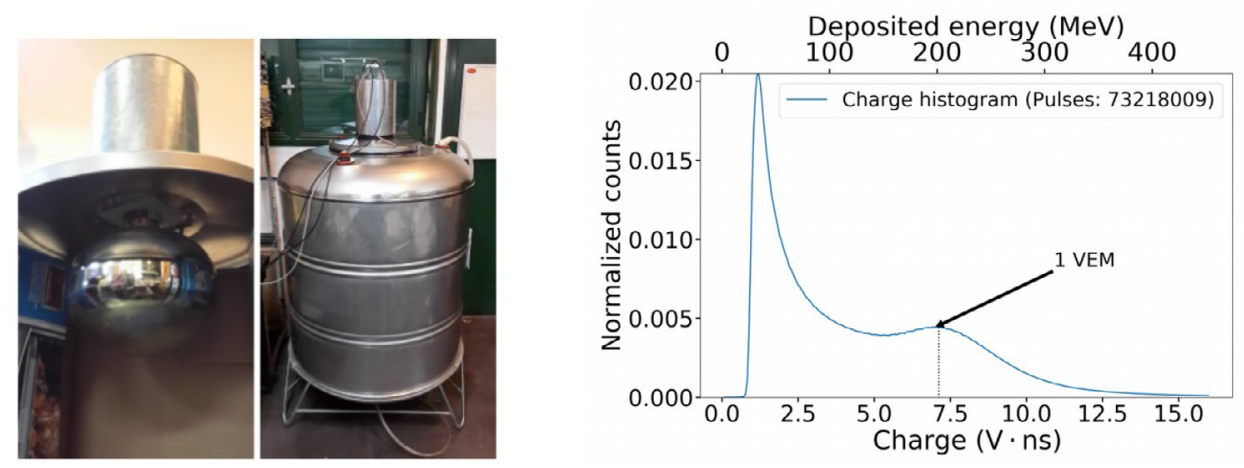

Figure 1: Left: Pictures of the Neurus WCD (from Dasso et al. 2015). Right: Calibrated charge histogram. 
Since 2019, and during the first year of operations, two parallel acquisition systems have been working. On one hand, an oscilloscope in the rate mode counts the pulses that exceed a peak threshold and then this rate is recorded by a communication system. The threshold was chosen in such a way as to detect SCRs with deposited energy associated with the electromagnetic component. These data will be presented in the next section. On the other hand, a Red Pitaya STEMlab board in oscilloscope mode records the trace of three sample pulses per second, limited by the acquisition speed of Red Pitaya and the communication with the computer. The area of each voltage pulse $V(t)$ (or charge) represents the energy deposited by each particle.

The charge histogram for pulses recorded during the first 10 months of the uninterrupted operation of the detector, between 25 March 2019 and 11 January 2020, is shown in the right panel of figure 1 . To generate this histogram, we consider 300 bins and the 73218009 pulses recorded. The first peak is related to the trigger threshold and is mainly generated by the electromagnetic component of the EAS. Intermediate values, evidenced by a characteristic peak called the muon hump, are dominated by single muons through the detector. Higher values of charge in the histogram correspond to simultaneous entrance of multiple particles to the detector called mini-showers (e.g., Asorey \& Dasso 2015). This second maximum corresponds to the deposited energy by vertical muons. The charge histogram can be re-interpreted as a function of the deposited energy by vertical muons, assuming that a single muon deposits $2 \mathrm{MeV} \mathrm{cm}^{-1}$ in water (e.g., Asorey 2011) and knowing that the water level in the tank is $100 \mathrm{~cm}$. With this calibration the value of 1 VEM (Vertical Equivalent Muon) in electronic units is obtain; in our case $1 \mathrm{VEM}=(200 \pm 10) \mathrm{MeV}$. This calibration enables the study of the time evolution of transient heliospheric events for different bands of deposited energy (e.g., Asorey 2011).

\section{SCRs corrected count rate}

The oscilloscope in the rate mode counts the total number of particles that enter the tank. The largest contribution to these rates corresponds to low-energy SCRs. Its average is around 160 counts per second. In this work we build the hourly average for the count rate $(S)$ and for the atmospheric variables for the same time period of the previous section.

The flux of low-energy SCRs at ground level is influenced by atmospheric conditions. It is essential to remove these effects before studying its relationship with space weather events. As a first approach, we performed a pressure correction that is based on a linear regression of $S$ and the surface pressure measurement $(P)$ :

$$
\frac{\Delta S}{S_{0}}=\beta \Delta P
$$

where $\Delta \mathrm{S}=\mathrm{S}-\mathrm{S}_{0}$ and $\Delta \mathrm{P}=\mathrm{P}-\mathrm{P}_{0} . \mathrm{S}_{0}$ and $\mathrm{P}_{0}$ are the mean count rate and pressure, respectively, during the time period considered and $\beta$ is the barometric coefficient, which depends on many factors, such as the nature of the secondary component and the altitude where the observation is performed (Dorman 2004).
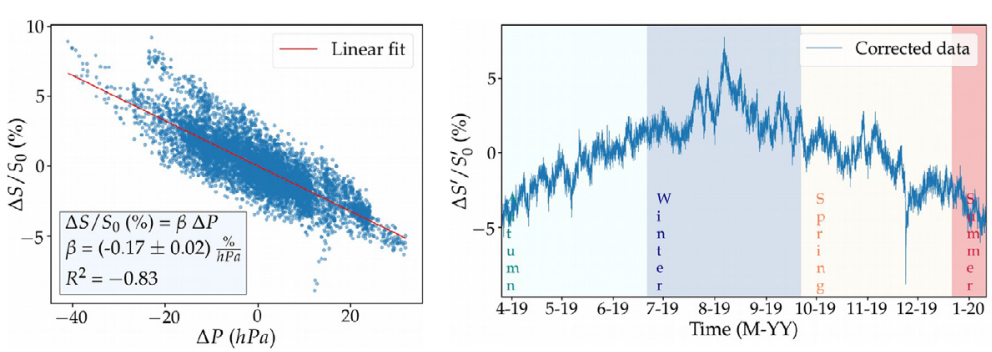

Figure 2: Left: Negative correlation between pressure deviation and the count rate relative deviation during the period considered. Right: Time series of the corrected count rate relative deviation for the same period. 
Left panel in figure 2 shows the negative correlation found between the count rate relative deviation and the atmospheric pressure variation. Using a least-squares fitting method, we found that the barometric coefficient in Marambio is $\beta=-(0.17 \pm 0.02) \% / \mathrm{hPa}$. After removing the pressure effect, a correction due to the indoor temperature was performed. Right panel in figure 2 shows the time series of $\Delta S^{\prime} / S_{0}^{\prime}$ (the count rate relative deviation corrected for pressure and indoor temperature). It is clear that it shows a seasonal modulation with a maximum in winter and a minimum in summer. This is consistent with what has been discussed in e.g., De Mendonça (2013) and Serap Tilav (2009), and it will be considered in the future to evaluate an adequate correction of this seasonal atmospheric effect.

\section{Comparison with neutron monitor}

We performed a preliminary comparison of Neurus with data from the Oulu neutron monitor $(65.05 \mathrm{~N}-25.47 \mathrm{E})$. The Oulu vertical geomagnetic rigidity cut off is about $0.8 \mathrm{GV}$ (Usoskin et al. 2001), similar and a bit lower than the one at Marambio. To be able to compare the time series, we remove the seasonal effect of the Neurus data and both data bases were normalized in such way that they have null mean and standard deviation equal to one. These two normalized time series for April 2019 are shown in figure 3. We see that they are in good agreement.

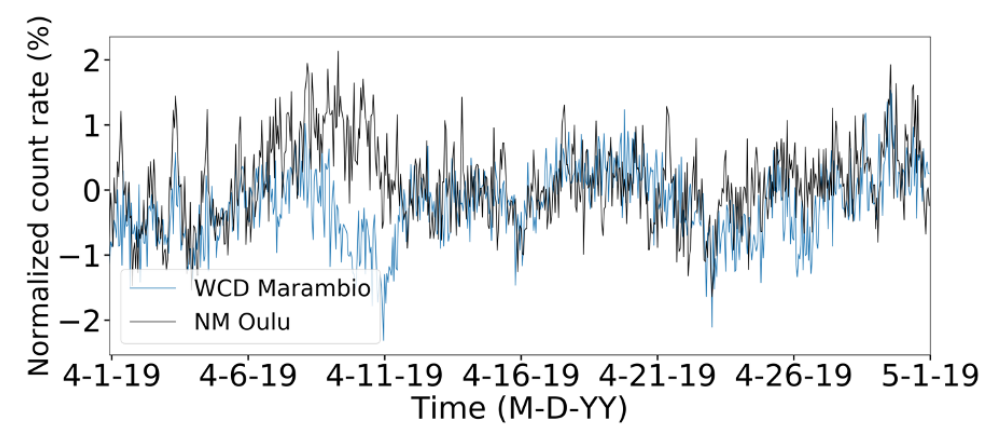

Figure 3: Normalized corrected count rate of Neurus WCD (blue line) compared to corrected count rate of Oulu NM (black line) for April 2019.

\section{Discussion and conclusion}

In this work we have presented a new cosmic ray detector for space weather studies based on Water Cherenkov effect, installed in the Antarctic Peninsula in 2019, as part of the LAGO detection network. We selected the first ten months of operation in order to calibrate its count rate. The expected anticorrelation between the count rate and the atmospheric pressure was corrected and we obtained a barometric coefficient: $\beta=-(0.17 \pm 0.02) \% / \mathrm{hPa}$. A seasonal modulation was observed. We present the calibration of the charge histogram of Neurus and also made a comparison between Neurus WCD and Oulu NM on April 2019, which results in good agreement.

Since the upgrade made at the beginning of 2020, we are recording the trace of all particles that reach the detector, and we are able to make deeper analysis of the Neurus measurements as for instance to analize the variability of the charge histogram with much higher time cadence. 


\section{Acknowledgments}

The authors acknowledge for the support from the Argentinean grants UBACYT (UBA) and PIP11220130100439 CO (CONICET). The LAGO collaboration is very grateful to all the participating institutions and The Pierre Auger collaboration for their continuous support. Acknowledgments to the NMDB database (https://www.nmdb.eu/), founded under the European Union's FP7 programme for providing data, as well as to the Oulu neutron monitor webpage at http://cosmicrays.oulu. fi and the Sodankyla Geophysical Observatory.

\section{References}

Allekotte, I., et al., 2007, Nucl. Instrum. Methods Phys. Res Section A 586, 409-420

Asorey, H., 2011, Proceedings of the 32nd International Cosmic Ray Conference (ICRC2011) 11, SH3-SH4, 467

Asorey, H., Dasso. S, 2015, Proceedings of the 34th International Cosmic Ray Conference - PoS (ICRC2015) 236, 247

Dasso, S., Asorey, H., 2012, Adv. Space Res 49, 1563-1569

Dasso, S., et al., 2015, Proceedings of the 34th International Cosmic Ray Conference - PoS (ICRC2015) 236, 105

De Mendonça, R. R. S., et al., 2013, J. Geophys. Res: Space Physics 118, 1403-1409

Dorman, L. I., 2004, Cosmic Rays In The Earth's Atmosphere And Underground, Dordrecht: Kluwer Acad. Publ.

Karle, A., 2009, Nucl. Instrum. Methods Phys. Res Section A 604, S46-S52

Masías-Meza, J. J., Dasso, S., 2014, Sun and Geosphere 9, 41-47

Sarmiento-Cano, C., et al., 2019, Proceedings of the 36th International Cosmic Ray Conference - PoS (ICRC2019) 358, 412

Sidelnik, I., 2016, Proceedings of the 34th International Cosmic Ray Conference - PoS (ICRC2015) 236, 665

The Pierre Auger collaboration, 2011, Journal of Instrumentation 6, 1003

Tilav, S., et al., 2010, Proceedings of the 31st International Cosmic Ray Conference (ICRC2009)

Usoskin, I.G, Mursula, K, Kangas, J., 2001, Proceedings of the 27th International Cosmic Ray Conference (ICRC2001), Copernicus Gesellschaft, 3842 